\title{
蛋白酶体亚基对肝细胞癌发生发展的调控作用 研究进展
}

\author{
胡靖依, 王青青, 刘 杨 \\ 浙江大学医学院免疫学研究所, 浙江 杭州 310058
}

\begin{abstract}
[摘 要] 蛋白酶体是真核细胞中负责降解细胞内短寿命蛋白、参与维持细胞内 蛋白质稳态的重要细胞器。研究表明, 在肝细胞癌 ( HCC) 的发生发展进程中, 蛋白 酶体调节颗粒亚基可通过调节PTEN基因、P53、Bcl-2、Bcl-2相互作用的细胞死亡 介体蛋白、周期蛋白依赖性激酶 $4 、 \beta$ 型转化生长因子受体、 $\mathrm{E} 2 \mathrm{~F} 1$ 、生长因子受体 结合蛋白 2 等多种肿瘤相关蛋白以及相关的通路分子, 例如信号转导及转录激活蛋 白3、蛋白激酶 $\mathrm{B}$, 诱使这些蛋白质功能失调, 进而促进 HCC的发生, 癌细胞增殖、 侵袭与转移。蛋白酶体核心颗粒亚基则更多参与HCC相关蛋白的降解, 因此核心 颗粒的抑制剂表现出良好的抗肿瘤效应。本文就当前蛋白酶体调节颗粒亚基和核 心颗粒亚基在HCC发生发展过程中的调控作用及其机制进行综述。
\end{abstract}

[关键词］蛋白酶体;蛋白酶体调节颗粒;蛋白酶体核心颗粒; 肝细胞癌;机制; 综述 [中图分类号 ］R735.7［文献标志码］A

\section{Research progress on proteasome subunits in regulating occurrence and development of hepatocellular carcinoma}

HU Jingyi, WANG Qingqing, LIU Yang (Institute of Immunology, Zhejiang University School of Medicine, Hangzhou 310058, China)

Corresponding author: LIU Yang, E-mail: liuyang0620@zju.edu.cn, https://orcid.org/ 0000-0001-9983-7019

\begin{abstract}
[ Abstract ] Proteasome is the eukaryotic organelle responsible for degradation of short-lived proteins and involved in maintaining cellular protein homeostasis. It has been reported that during the occurrence and development of hepatocellular carcinoma (HCC), the regulatory particle subunits of proteasome regulate a series of tumor-related proteins, and proliferation, survival-associated signaling molecules, including PTEN gene, P53, Bcl-2, Bcl-2 interacting mediator of cell death (Bim), cyclin-dependent kinase 4(CDK4),
\end{abstract}

收稿日期:2021-01-11 接受日期:2021-04-24 网络预发表日期:2021-05-27

基金项目:国家自然科学基金(81872248)

第一作者:胡靖依,硕士研究生,主要从事肿瘤免疫与抗病毒天然免疫研究;E-mail :hjy558@zju.edu.cn; https ://orcid.org/ 0000-0002-8708-4037

通信作者:刘 杨, 副教授,硕士生导师,主要从事肿瘤免疫与抗病毒天然免疫研究;E-mail : liuyang0620@zju.edu.cn; https ://orcid.org/0000-0001-9983-7019 
transforming growth factor $\beta$ receptor (TGFBR), E2F1, growth factor receptor-bound protein 2 (GRB2) . Meanwhile, these subunits regulate some tumor-associated pathway protein, such as signal transducer and activator of transcription 3 (STAT3) and protein kinase B (AKT), inducing their malfunction to promote the occurrence, proliferation, invasion and metastasis of HCC. The core particle subunits are more to perform the degradation of HCC-related proteins, so inhibitors targeting the core particle show a good anti-tumor effect. This review summarizes the current research progress on the regulation and mechanism of proteasome subunits in promoting the occurrence and development of HCC.

[ Key words ] Proteasome; Proteasome regulatory particle; Proteasome core particle; Hepatocellular carcinoma; Mechanism; Review

[J Zhejiang Univ (Med Sci), 2021, 50(3): 396-402.]

[缩略语] 肝细胞癌 (hepatocellular carcinoma, HCC); 调节颗粒非ATP酶亚基 (regulatory particle non-ATPase, Rpn); $\beta$ 型转化生长因子受体 (transforming growth factor $\beta$ receptor, TGFBR); 小窝蛋白 (caveolin, CAV); 生长因子受体结合蛋白 (growth factor receptor-bound protein,GRB); 磷脂酰肌醇3激酶/蛋白激酶B(phosphoinositide 3-kinase) kinase B,PI3K/AKT); Bcl-2相互作用的细胞死亡介体蛋白 (Bcl-2 interacting mediator of cell death,Bim); 调节颗粒AAA ATP酶(regulatory particle triple ATPase,Rpt); 腺苷三磷 酸 (adenosine triphosphate, ATP); 缺氧诱导因子(hypoxia-inducible factor, HIF); 环氧合 酶(cyclooxygenase, COX); 磷酸化肉瘤酪氨酸激酶(phosphorylated sarcoma tyrosine kinase,p-SRC); Bcl-2相关X蛋白 (Bcl-2 associated X protein, BAX); 周期蛋白依赖性激酶 (cyclin-dependent kinase, CDK); 肉瘤酪氨酸激酶同源区2-蛋白酪氨酸磷酸酶( sarcoma tyrosine kinase homology domain 2- containing protein tyrosine phos-phatase, SHP); 信号 转导及转录激活蛋白 (signal transducer and activator of transcription,STAT); 自噬相关基 因 (autophagy related gene,ATG);20S蛋白酶体 $\alpha$ 亚基(proteasome $20 \mathrm{~S}$ subunit alpha, PSMA );20S蛋白酶体 $\beta$ 亚基 (proteasome 20S subunit beta,PSMB)

蛋白酶体是细胞内重要的亚器结构, 密度梯 度离心时的沉降系数为 $26 \mathrm{~S}$, 因此被称为 $26 \mathrm{~S}$ 蛋白酶 体。26S蛋白酶体由20S核心颗粒以及结合在核心 颗粒两端的19S调节颗粒组成 ${ }^{[1]}$ 。蛋白酶体不仅介 导错误折叠、老化或氧化损伤的异常蛋白质的清 除, 还广泛影响细胞信号转导、周期、增殖等生命活 动。研究表明, 蛋白酶体参与调节与细胞周期进程 及致癌作用相关的多种关键蛋白的代谢过程, 并在 如原癌基因蛋白、抑癌蛋白以及相关转录因子和信 号分子的降解过程中发挥重要作用 ${ }^{[2]}$ 。

肝癌的细胞来源多样, 临床上绝大部分病例 为肝细胞来源的肝癌, 即 $\mathrm{HCC}^{[3]}$ 。 $\mathrm{HCC}$ 发生过程 涉及多种基因的突变以及多种突变类型, 如染色 体缺失、插人、倒位等, 这些突变因素造成肿瘤细
胞内基因拷贝数高于正常细胞,促使肿瘤细胞内 蛋白合成水平升高 ${ }^{[4]}$ 。因而, 相比于正常细胞, HCC 细胞具有更高的蛋白酶体拷贝水平与活性, 以维持细胞内蛋白质水平的稳定 ${ }^{[5]}$ 。在一定程度 上, 肿瘤细胞的生存、增殖、侵袭和转移等生命活 动与蛋白酶体的功能有着紧密的联系 ${ }^{[6]}$ 。因此, 抑制蛋白酶体的活性成为肿瘤化疗的一种思路。 有研究结果显示, 蛋白酶体抑制剂与肿瘤常规化 疗药物联合使用可以增强化疗效果 ${ }^{[7-9]}$ 。但在临 床上,蛋白酶体抑制剂尚未广泛应用于HCC治 疗。本文对参与HCC进程的蛋白酶体调节颗粒和 核心颗粒亚基的具体功能及机制进行综述, 并对 蛋白酶体在HCC发生发展中的研究方向及临床应 用进行展望。 


\section{1 蛋白酶体调节颗粒亚基促进HCC的发生发展}

19S调节颗粒由 “盖子” (lid)与 “底座” (base) 组成。调节颗粒的主要作用是识别泛素化的底物 蛋白, 催化蛋白的去泛素化修饰、去折叠等, 为目 标蛋白进入蛋白酶体核心颗粒做准备 ${ }^{[10]}$ 。在此 过程中, 调节颗粒对HCC多种相关蛋白的降解有 着不同程度的影响。

\section{1 调节颗粒 “盖子” 促进HCC的发生发展}

调节颗粒的 “盖子” 由9个非ATP酶蛋白组 成, 分别为 Rpn3、Rpn5、Rpn6、Rpn7、Rpn8、Rpn9、 $R p n 11 、 R p n 12$ 和Rpn15, 目前仅发现Rpn11参与 HCC的调控过程。Rpn11是一种去泛素化酶, 催化 底物蛋白的去泛素化过程, 在蛋白酶体对底物蛋 白的降解过程中发挥关键作用 ${ }^{[11]}$ 。Wang 等 ${ }^{[12]}$ 发 现, Rpn11通过去除TGFBR上的K63泛素链,抑制 TGFBR降解, 进而增强HCC细胞中 $\beta$ 型转化生长因 子信号转导过程, 而在缺乏Rpn11的小鼠肝细胞 中,TGFBR蛋白水平明显降低。TGFBR也可经溶 酶体途径降解, 由CAV1介导, 将TGFBR向溶酶体 高效转运 ${ }^{[13]}$ 。Rpn11催化CAV1的去泛素化修饰, 减弱CAV1介导的TGFBR1和TGFBR 2 经溶酶体途 径的降解过程 ${ }^{[12]}$, 在一定程度上加剧了 HCC的恶 性转移(图1)。Rpn11也可通过催化E2F1的去泛 素化过程,使E2F1因子免于蛋白酶体降解而稳定 存在于细胞内 ${ }^{[14]}$ 。临床证据显示, E2F1异常上调 常见于各种类型的肿瘤, 且与肿瘤的恶性进展和 患者不良生存预后相关 ${ }^{[14]}$ 。Rpn11可通过上调 $\mathrm{E} 2 \mathrm{~F} 1$ 水平, 增强 $\mathrm{E} 2 \mathrm{~F} 1$ 下游的细胞生存信号, 上调 存活蛋白Survivin和叉头框蛋白M1等多种蛋白表 达, 促进裸鼠 HCC 的发生与发展 ${ }^{[14]}$ (图 1)。 Lv 等 ${ }^{[15]}$ 还发现Rpn11为GRB2 的新型翻译后修饰因 子。GRB2在多种类型的肿瘤中均具有促进肿瘤 侵袭和转移的作用。Rpn11可催化GRB2去泛素化 修饰, 使其免于蛋白酶体降解, 并在HCC细胞内持 续存在并累积, 导致PI $3 \mathrm{~K} / \mathrm{AKT}$ 信号通路持续激 活, 最终促进下游的细胞增殖、侵袭和转移等通 路, 抑制Rpn 11 则可有效抑制 HCC 细胞的恶性行 为 ${ }^{[15]}$ 。此外, Rpn11可对P53和Bim进行去泛素化 修饰从而抑制其降解, 抵抗细胞调亡, 从而促进 $\mathrm{HCC}$ 的发生 ${ }^{[16]}$ (图1)。

\section{2 调节颗粒 “底座” 促进HCC的发生发展} 调节颗粒 “底座” 含有一个由 6 个不同的
$\mathrm{Rpt}$ 组成的六聚杂环。这些亚基分别被命名为 Rpt1 6。Rpt利用ATP结合和水解的能量对底物蛋 白施加拉力, 将其展开并通过狭窄的中央孔将多 肽转运到核心颗粒的肽酶腔中 ${ }^{[17]}$ 。“底座” 除 了上述Rpt组成的六聚杂环外, 还包含 4 个非ATP酶 蛋白，分别为Rpn1、Rpn2、Rpn10和Rpn13。其中， Rpn1、Rpn10和Rpn13是泛素分子受体，可通过不 同的位点识别、结合泛素分子,或直接识别并结合 含有泛素样结构域的底物蛋白 ${ }^{[18]}$ 。此外，“底 座” 还包含癌针蛋白重复序列伴侣蛋白 (Gankyrin) 等若干与蛋白酶体组装相关的伴侣蛋白。这些伴 侣蛋白通过与对应的 “底座” Rpt 相互作用,协助 蛋白酶体的装配并维持其稳定性 ${ }^{[19]}$ 。

1.2.1Rpn1和Rpn2通过增强细胞内脂质代谢促 进HCC细胞增殖与转移 Rpn1和Rpn2是 $26 \mathrm{~S}$ 蛋白 酶体调节颗粒 “底座” 中的两个非ATP酶亚基,其 中Rpn1为泛素分子受体。Rpn1和Rpn2可通过调 节细胞周期进程影响胃癌、乳腺癌等多种肿瘤细 胞增殖 ${ }^{[20-22]}$ 。同时, Rpn1和Rpn2也属于低密度脂 蛋白相关蛋白,参与脂类的代谢过程。Tan等 ${ }^{[23]}$ 发现, Rpn1和Rpn2可通过上调脂质和脂肪酸合成 相关基因促进HCC细胞内的脂质代谢过程，从而 促进细胞增殖。Rpn1和Rpn2在细胞质中可结合 于脂滴的表面, 其在HCC细胞中高表达可促进细 胞内脂肪酸和脂滴的堆积, 这种高脂的细胞内环 境促进了 $\mathrm{HCC}$ 细胞的增殖和转移 ${ }^{[23]}$ 。临床证 据也表明两者的高表达与HCC患者不良预后密切 相关 ${ }^{[23]}$ 。

1.2.2Rpn10通过促进多种抑癌蛋白降解促进 HCC 细胞增殖 $26 \mathrm{~S}$ 蛋白酶体识别多聚泛素链是 细胞选择性降解靶蛋白的关键步骤。Rpn10是蛋 白酶体中主要的泛素受体之一, 与多聚泛素链有 非常强的结合能力 ${ }^{[24]}$ 。临床研究表明,Rpn10高 表达与HCC患者的不良预后直接相关 ${ }^{[25]}$ 。Rpn10 可直接促进HCC细胞中的PTEN基因经泛素-蛋白 酶体途径降解, 从而抑制PTEN-PI3K/AKT信号通 路转导, 造成HCC细胞的增殖愈加活跃 ${ }^{[25]}$ 。另外, HCC作为一种实体瘤,瘤体中心缺氧较为严重, 因 此HIF- $1 \alpha$ 可在细胞内累积, 且HIF- $1 \alpha$ 可以直接结 合到Rpn10的启动子区域 ${ }^{[25]}$, 这也从另一个角度 解释了Rpn10在HCC中高表达的现象。

研究发现,沉默Rpn10可抑制 HCC 细胞增殖 和肿瘤生长, 促进肿瘤细胞凋亡 ${ }^{[26]}$ 。COX2、p-SRC 


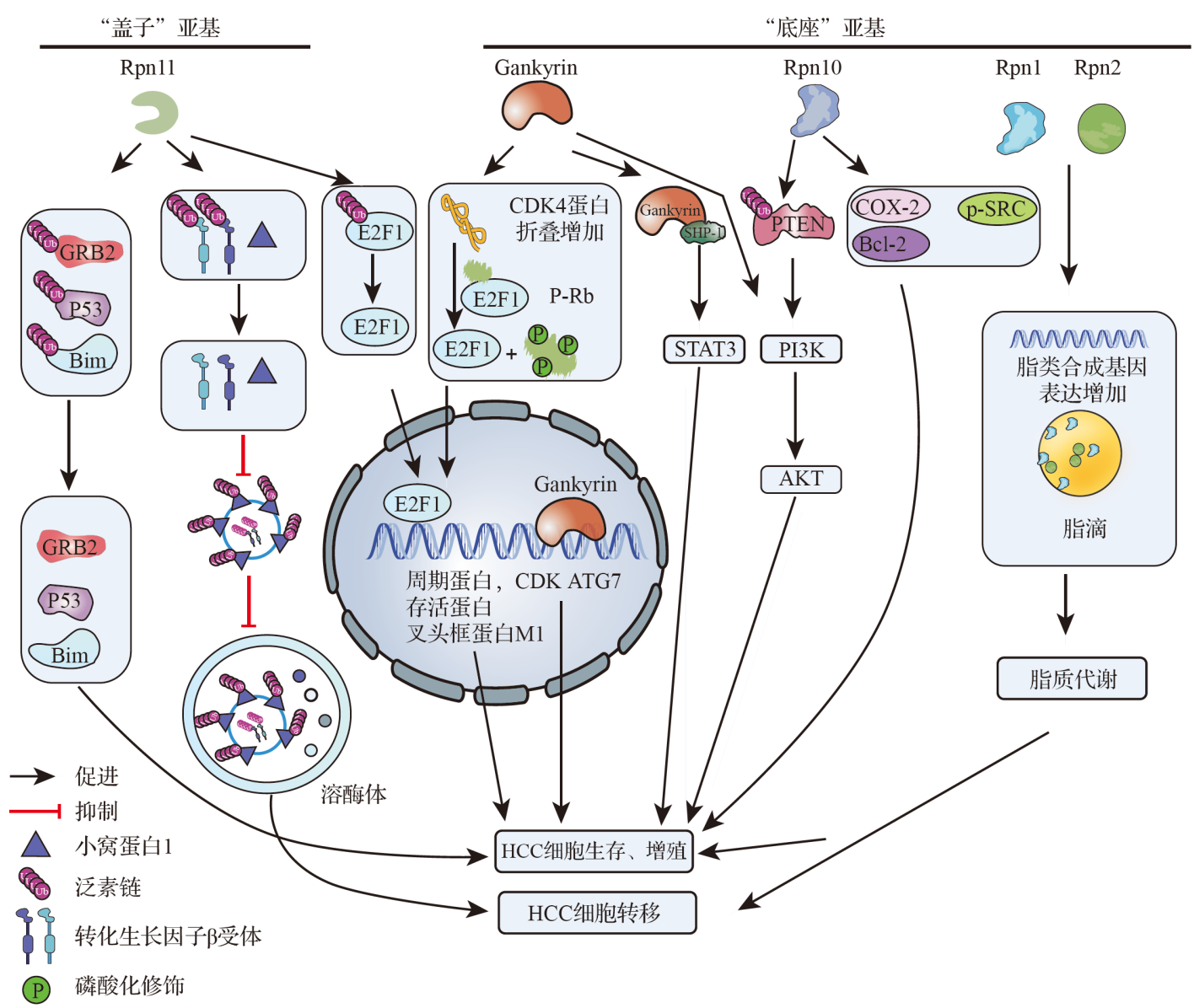

在肝细胞癌(HCC)细胞内, 蛋白酶体调节颗粒亚基通过不同的机制发挥作用. “盖子” 亚基: 调节颗粒非ATP酶亚基(Rpn)11通过 对生长因子受体结合蛋白 2(GRB2)、P53、Bcl-2相互作用的细胞死亡介体蛋白 (Bim) 以及E2F1的去泛素化修饰, 不断激活细胞内的存 活和增殖信号, 促进HCC的发生. 其中, Rpn11对小窝蛋白和 $\beta$ 型转化生长因子受体 (TGFBR)的去泛素化修饰抑制了网格蛋白介导的 对TGFBR的内吞作用, 进而抑制TGFBR经溶酶体途径的降解, 使TGFBR持续发挥作用, 从而促进HCC的转移. “底座” 亚基: (1)Gankyrin直接扰乱了周期蛋白依赖性激酶 4(CDK4) 的折叠进程, 造成大量CDK4在细胞质内累积, 直接催化结合了E2F1的视网膜细胞瘤 基因蛋白 $(\mathrm{Rb})$ 的磷酸化, $\mathrm{Rb}$ 磷酸化后释放出 $\mathrm{E} 2 \mathrm{~F} 1$ 人核, 直接诱导细胞周期相关基因的转录, 促进HCC细胞增殖; Gankyrin也可以自身 作为一种转录因子, 转位人核诱导自噬相关基因7 (ATG7)的转录, 增强HCC细胞的自噬功能, 进而促进肿瘤细胞存活; Gankyrin还可 以与肉瘤酪氨酸激酶同源区2-蛋白酪氨酸磷酸酶 1 (SHP-1)相互作用, 活化信号转导及转录激活蛋白 3 (STAT3) 通路, 或直接活化磷脂 酰肌醇3激酶/蛋白激酶B $\mathrm{PI} 3 \mathrm{~K} / \mathrm{AKT}$ )信号通路, 促进HCC增殖. (2)Rpn10不仅可以上调癌基因蛋白环氧合酶2(COX-2)、Bcl-2和磷酸 化肉瘤酪氨酸激酶(p-SRC)的表达, 还可促进抑癌基因蛋白PTEN的降解. 3Rpn1和Rpn2则可通过增强细胞脂质代谢促进HCC发生、 癌细胞增殖和转移.

图 1 蛋白酶体调节颗粒亚基在HCC中的作用机制

Figure 1 Mechanisms of proteasome regulatory particles subunits in HCC

和 $B c l$-2 为促进肿瘤恶性行为的经典基因, $P 53$ 和 $B A X$ 为典型促细胞调亡基因,沉默 $R p n 10$ 基因可显 著抑制COX2、p-SRC、Bcl-2等原癌基因的表达, 增 加P53和BAX等抑癌基因的表达(图1), 提示Rpn10 在HCC中起着原癌基因的作用。

1. 2.3 Rpn13促进HCC细胞增殖 Rpn13(又称 黏附调节分子 1 ) 作为泛素受体而发挥作用 ${ }^{[24]}$ 。 研究发现, Rpn13 可促进HCC 细胞增殖, 可能与 HCC 的发生有关, 但具体机制有待深人研究 ${ }^{[27]}$ 。 新型蛋白酶体抑制剂双亚茮基哌啶RA190通过结 合Rpn13、抑制核因子 $\kappa$ B 通路诱导HCC细胞凋亡,
与索拉菲尼在体外产生协同抗肿瘤作用, 并可降 低HCC模型小鼠的肿瘤增殖 ${ }^{[28]}$ 。

1.2. $4 R p t 1$ 和Rpt2参与 HCC调控 Rpt 1 和Rpt2 为调节颗粒 “底座” 上的Rpt亚基之一。在ATP存 在的情况下,Rpt的羒基端会与核心颗粒 $\alpha$ 亚基环 $(\alpha 1 \sim \alpha 7)$ 上的特异性位点结合, 从而触发并开放门 控通道, 促进底物进入肽酶腔中 ${ }^{[29]}$ 。Rpt1在多种 肿瘤中均发挥原癌基因的作用 ${ }^{[30]}$ 。在HCC中, Rpt 1 可上调细胞周期蛋白 D 1 的表达水平,诱导 HCC 的发生和癌细胞增殖 ${ }^{[31]}$ 。在多发性骨髓瘤 中, Rpt2缺失可增强肿瘤对嗍替佐米耐药, 且硼替 
佐米可稳定细胞内的 $R p t 2^{[32-33]}$ 。而在乙型肝炎病 毒感染的HCC细胞中, Rpt2可能作为一种转录抑 制因子抑制乙型肝炎病毒基因的转录 ${ }^{[34]}$ 。Cui 等 ${ }^{[35]}$ 研究发现小鼠的HCC细胞中Rpt2表达上调。 这些研究均提示Rpt2可能抑制HCC的发展, 但具 体的机制有待进一步研究。

1. 2. 5 Gankyrin促进HCC细胞增殖、侵袭及转 移 Gankyrin为蛋白酶体调节颗粒 “底座” 上的 伴侣蛋白, 其高度保守且含有7个针蛋白重复序 列, 在蛋白酶体的组装过程中发挥重要作用。 Gankyrin也是一种重要的癌蛋白, 在多种肿瘤中表 达增加, 在肿瘤的发生和发展中起到关键作 用 ${ }^{[36]}$ 。Gankyrin可与CDK4作用, 加速CDK4的正 常折叠过程, 导致大量CDK4释放到细胞质中, 催 化视网膜细胞瘤基因蛋白磷酸化, 并释放与其结 合的 $\mathrm{E} 2 \mathrm{~F} 1$ 转录因子, 促进 $\mathrm{S}$ 期所必需的细胞周期蛋 白和 $C D K$ 等基因的转录, 从而诱导细胞从 $\mathrm{G} 1$ 期进 人S期, 促进HCC细胞增殖 ${ }^{[37]}$ (图1), 抑制Gankyrin 可显著抑制HCC的进程 ${ }^{[38]}$ 。

Gankyrin还可与SHP1结合, 通过增强STAT3 活化直接诱导 HCC 的发生; 在Gankyrin缺陷小鼠 中, STAT3的活化程度减弱, 而肿瘤干细胞表面标 志分子表达水平也较低 ${ }^{[36,39]}$ (图1)。Gankyrin还 可通过增强PI $3 \mathrm{~K} / \mathrm{AKT} / \mathrm{HIF}-1 \alpha$ 信号轴下游的基 因, 如血管内皮生长因子的表达, 促进HCC的侵袭 及转移 ${ }^{[39-40]}$ 。

作为蛋白酶体调节颗粒亚基之一, 在HCC细 胞处于饥饿或应激状态时, Gankyrin可转位人核, 协同热休克转录因子 $1 、 A T G 7$ 的启动子区域结合, 上调 $A T G 7$ 基因的表达, 增强肿瘤细胞的自噬过 程,使细胞免于饥饿或应激导致的损伤及死亡 ${ }^{[41]}$ (图1)。临床上Gankyrin导致的HCC患者对化疗 药物耐药或许与这种机制有关。同时, 细胞氧化 应激也可升高Gankyrin的表达水平, 通过核因子 E2 相关因子 2 的负反馈调节来发挥抗氧化应激 效应, 从另一个方面促进了肿瘤细胞的生存 ${ }^{[42]}$ 。

在细胞代谢层面, Gankyrin 可通过激活 $\beta$-catenin/c-Myc通路来促进糖酵解和谷氨酰胺 分解, 极大地增强了瓦博格效应。研究显示, 高 表达Gankyrin的HCC细胞中葡萄糖的摄取、糖 酵解速率和乳酸水平更高 ${ }^{[43]}$, 这表明 Gankyrin 可从能量方面为 HCC 细胞的行为提供强有力的 后盾 ${ }^{[44]}$ 。

\section{2 蛋白酶体核心颗粒亚基促进 HCC的发生发展}

蛋白酶体核心颗粒为一筒状圆柱体结构, 是 蛋白质降解的场所, 由 $\alpha$ 亚基与 $\beta$ 亚基组成, 每个平 面分别由 7 个不同的 $\alpha$ 或 $\beta$ 亚基组成七聚杂环, 最终 成为一个 $\alpha_{1-7}-\beta_{1-7}-\beta_{1-7}-\alpha_{1-7}$ 的四层结构。核心颗粒 的7个 $\alpha$ 亚基分别由PSMA1 7基因编码, 目前关于 其功能知之甚少,一般认为在协同 $\beta$ 亚基的催化活 动中发挥作用 ${ }^{[45]}$ 。 $\beta$ 亚基由PSMB1 7基因编码, 其中的 $\beta 1 、 \beta 2 、 \beta 5$ 具有不同的蛋白酶催化特性,分 别表现为类胱天蛋白酶样活性、酪氨酸蛋白酶活 性、胰凝乳蛋白酶样活性 ${ }^{[46]}$ 。在靶蛋白进人蛋白 酶体内部的过程中,蛋白酶体的调节颗粒负责靶 蛋白的选择,而核心颗粒则执行对靶蛋白的降解, 进人核心颗粒的蛋白质最终都被水解成2 24个氨 基酸大小的肽段 ${ }^{[47]}$ 。

目前核心颗粒亚基与HCC直接相关的研究相 对较少。在小鼠的HCC中,核心颗粒亚基PSMAG 与PSMB 4 表达均上调, 提示核心颗粒亚基参与 HCC 的调控过程 ${ }^{[35]}$ 。Qin等 ${ }^{[48]}$ 研究发现, HCC 肿 瘤组织间液中的PSM A 1 水平显著降低, 认为 PSMA1具有作为HCC的诊断标志的潜力。PSMB7 可促进 HCC 细胞增殖及其对 5 -氟尿嘧啶的耐 药 ${ }^{[49]}$ 。大量临床研究证实, 抑制蛋白酶体核心颗 粒活性可有效抑制HCC进程。作为一种可逆的特 异性抑制PSMB1和PSMB5的蛋白酶体抑制剂, 硼 替佐米可有效抑制HCC细胞增殖及诱导肿瘤细胞

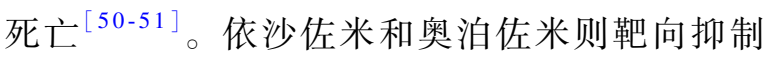
PSMB5, 两者的运用也可显著抑制HCC的活动进 程 ${ }^{[52-53]}$ 。上述研究结果提示, 核心颗粒作为降解 蛋白质的直接执行者, 在HCC进程的调控中有着 不可或缺的作用,蛋白酶体抑制剂在HCC的临床 治疗上可能具有重要的应用价值。

\section{3 结 语}

蛋白酶体可通过调节参与HCC进程中关键蛋 白的降解或表达、促进 HCC 细胞代谢以及增强 HCC 细胞自噬过程来促进HCC的发生发展。研究 提示, 抑制蛋白酶体的功能在HCC的治疗应用具 有较好的前景。目前针对蛋白酶体核心颗粒的一 些抑制剂在HCC的治疗上已经崭露头角, 而针对 蛋白酶体调节颗粒亚基的抑制剂仍需进一步探 索。不足的是, 蛋白酶体与HCC的相关研究大多 
仍停留在细胞和小鼠模型水平, 缺乏有力的临床 证据, 且对于蛋白酶体各亚基是如何与 HCC相关 蛋白相互作用的机制并未阐明。因此,蛋白酶体 对HCC的调控作用及其机制亟待更广泛、更系统 和更深人的研究, 以期为蛋白酶体抑制剂治疗 HCC 提供更为充分的理论依据。

\section{利益冲突 所有作者均声明不存在利益冲突}

\section{参考文献}

[1] BARD J A M, GOODALL E A, GREENE E R, et al. Structure and function of the $26 \mathrm{~S}$ proteasome $[\mathrm{J}]$. Annu Rev Biochem, 2018, 87(1): 697-724.

[2] COUX O, TANAKA K, GOLDBERG A L. Structure and functions of the $20 \mathrm{~S}$ and $26 \mathrm{~S}$ proteasomes $[\mathrm{J}]$. Annu Rev Biochem, 1996, 65(1): 801-847.

[3] BRAY F, FERLAY J, SOERJOMATARAM I, et al. Global cancer statistics 2018: GLOBOCAN estimates of incidence and mortality worldwide for 36 cancers in 185 countries[J]. CA Cancer J Clin, 2018, 68(6): 394-424.

[4] WILLIAMS B R, PRABHU V R, HUNTER K E, et al. Aneuploidy affects proliferation and spontaneous immortalization in mammalian cells $[\mathrm{J}]$. Science, 2008, 322(5902): 703-709.

[5] DESHAIES R J. Proteotoxic crisis, the ubiquitinproteasome system, and cancer therapy $[\mathrm{J}]$. BMC Biol, 2014, 12(1): 94.

[6] A three-drug combo for multiple myeloma[J]. Cancer Discov, 2016, 6(11): Of4.

[7] MOREAU P, MASSZI T, GRZASKO N, et al. Oral ixazomib, lenalidomide, and dexamethasone for multiple myeloma[J]. N Engl J Med, 2016, 374(17): 1621-1634.

[8] LUDWIG H, POENISCH W, KNOP S, et al. IxazomibThalidomide-Dexamethasone for induction therapy followed by Ixazomib maintenance treatment in patients with relapsed/refractory multiple myeloma $[\mathbf{J}]$. Br J Cancer, 2019, 121(9): 751-757.

[9] HOU J, JIN J, XU Y, et al. Randomized, double-blind, placebo-controlled phase III study of ixazomib plus lenalidomide-dexamethasone in patients with relapsed/ refractory multiple myeloma: China continuation study[J]. J Hematol Oncol, 2017, 10(1): 137.

[10 ] THROWER J S, HOFFMAN L, RECHSTEINER M, et al. Recognition of the polyubiquitin proteolytic signal $[\mathbf{J}]$. EMBO J, 2000, 19(1): 94-102.

[11] VERMA R, ARAVIND L, OANIA R, et al. Role of Rpn11 metalloprotease in deubiquitination and degradation by the $26 \mathrm{~S}$ proteasome $[\mathrm{J}]$. Science, 2002, 298(5593): 611-615.

[12] WANG B, XU X, YANG Z, et al. POH1 contributes to hyperactivation of TGF- $\beta$ signaling and facilitates hepatocellular carcinoma metastasis through deubiquitinating TGF- $\beta$ receptors and caveolin-1[J].
EBioMedicine, 2019, 41: 320-332.

[13] CHEN Y G. Endocytic regulation of TGF- $\beta$ signaling $[\mathrm{J}]$. Cell Res, 2009, 19(1): 58-70.

[14] WANG B, MA A, ZHANG L, et al. POH1 deubiquitylates and stabilizes E2F1 to promote tumour formation $[J]$. Nat Commun, 2015, 6(1): 8704.

[15] LV J, ZHANG S, WU H, et al. Deubiquitinase PSMD14 enhances hepatocellular carcinoma growth and metastasis by stabilizing GRB2 $[\mathrm{J}]$. Cancer Lett, 2020, 469: 22-34.

[16] WANG C H, LU S X, LIU L L, et al. POH1 knockdown induces cancer cell apoptosis via p53 and $\operatorname{bim}[\mathrm{J}]$. Neoplasia, 2018, 20(5): 411-424.

[17] OLIVARES A O, BAKER $T$ A, SAUER $R \mathrm{~T}$. Mechanical protein unfolding and degradation [J]. Annu Rev Physiol, 2018, 80(1): 413-429.

[18] MARTINEZ-FONTS K, DAVIS C, TOMITA T, et al. The proteasome 19S cap and its ubiquitin receptors provide a versatile recognition platform for substrates $[\mathrm{J}]$. Nat Commun, 2020, 11(1): 477.

[19] BUDENHOLZER L, CHENG C L, LI Y, et al. Proteasome structure and assembly $[\mathrm{J}]$. J Mol Biol, 2017, 429(22): 3500-3524.

[20] XIONG W, WANG W, HUANG H, et al. Prognostic significance of PSMD1 expression in patients with gastric cancer[J]. J Cancer, 2019, 10(18): 4357-4367.

[21] OKUMURA T, IKEDA K, UJIHIRA T, et al. Proteasome 26S subunit PSMD1 regulates breast cancer cell growth through p53 protein degradation $[\mathrm{J}]$. J Biochem, 2018, 163(1): 19-29.

[22] LI Y, HUANG J, ZENG B, et al. PSMD2 regulates breast cancer cell proliferation and cell cycle progression by modulating $\mathrm{p} 21$ and $\mathrm{p} 27$ proteasomal degradation $[J]$. Cancer Lett, 2018, 430: 109-122.

[23] TAN Y, JIN Y, WU X, et al. PSMD1 and PSMD2 regulate HepG2 cell proliferation and apoptosis via modulating cellular lipid droplet metabolism $[\mathrm{J}]$. BMC Mol Biol, 2019, 20(1): 24.

[24] COLLINS G A, GOLDBERG A L. The logic of the 26s proteasome[J]. Cell, 2017, 169(5): 792-806.

[25] JIANG Z, ZHOU Q, GE C, et al. Rpn10 promotes tumor progression by regulating hypoxia-inducible factor 1 alpha through the PTEN/Akt signaling pathway in hepatocellular carcinoma[J]. Cancer Lett, 2019, 447: 1-11.

[26] CAI M J, CUI Y, FANG M, et al. Inhibition of PSMD4 blocks the tumorigenesis of hepatocellular carcinoma $[\mathrm{J}]$. Gene, 2019, 702: 66-74.

[27] YANG X, MIAO X, WEN Y, et al. A possible connection between adhesion regulating molecule 1 overexpression and nuclear factor kappa B activity in hepatocarcinogenesis $[J]$. Oncol Rep, 2012, 28(1): 283-290.

[28] SOONG R S, ANCHOORI R K, RODEN R B S, et al. Bis-benzylidine piperidone RA190 treatment of hepatocellular carcinoma via binding RPN13 and inhibiting 
NF-кB signaling [J ]. BMC Cancer, 2020, 20(1): 386.

[29] RABL J, SMITH D M, YU Y, et al. Mechanism of gate opening in the $20 \mathrm{~S}$ proteasome by the proteasomal ATPases[J]. Mol Cell, 2008, 30(3): 360-368.

[30] QIN J, WANG W, AN F, et al. PSMC2 is up-regulated in pancreatic cancer and promotes cancer cell proliferation and inhibits apoptosis $[\mathrm{J}]$. J Cancer, 2019, 10(20): 4939-4946.

[31] LIU Y, CHEN H, LI X, et al. PSMC2 regulates cell cycle progression through the $21 /$ cyclin $\mathrm{d} 1$ pathway and predicts a poor prognosis in human hepatocellular carcinoma[J]. Front Oncol, 2021, 11: 607021.

[32] SHI C X, KORTÜM K M, ZHU Y X, et al. CRISPR genome-wide screening identifies dependence on the proteasome subunit psmc6 for bortezomib sensitivity in multiple myeloma[J]. Mol Cancer Ther, 2017, 16(12): 2862-2870.

[33] YU T, TAO Y, YANG M, et al. Profiling human protein degradome delineates cellular responses to proteasomal inhibition and reveals a feedback mechanism in regulating proteasome homeostasis $[\mathrm{J}]$. Cell Res, 2014, 24(10): 1214-1230.

[34] ZHANG Z, TORII N, FURUSAKA A, et al. Structural and functional characterization of interaction between hepatitis $B$ virus $X$ protein and the proteasome complex $[\mathrm{J}]$. J Biol Chem, 2000, 275(20): 15157-15165.

[35] CUI F, WANG Y, WANG J, et al. The up-regulation of proteasome subunits and lysosomal proteases in hepatocellular carcinomas of theHBx gene knockin transgenic mice $[\mathrm{J}]$. Proteomics, 2006, 6(2): 498-504.

[36] FUJITA J, SAKURAI T. The oncoprotein gankyrin/ PSMD10 as a target of cancer therapy $[J]$. Adv Exp Med Biol, 2019, 1164: 63-71.

[37] DAWSON S, APCHER S, MEE M, et al. Gankyrin is an ankyrin-repeat oncoprotein that interacts with CDK4 kinase and the S6 ATPase of the $26 \mathrm{~S}$ proteasome $[\mathrm{J}]$. J Biol Chem, 2002, 277(13): 10893-10902.

[38] JIANG Y, IAKOVA P, JIN J, et al. Farnesoid X receptor inhibits gankyrin in mouse livers and prevents development of liver cancer[J]. Hepatology, 2013, 57(3): 1098-1106.

[39] SAKURAI T, YADA N, HAGIWARA S, et al. Gankyrin induces STAT 3 activation in tumor microenvironment and sorafenib resistance in hepatocellular carcinoma[J]. Cancer Sci, 2017, 108(10): 1996-2003.

[40] FU J, CHEN Y, CAO J, et al. p28GANK overexpression accelerates hepatocellular carcinoma invasiveness and metastasis via phosphoinositol 3-kinase/ AKT/hypoxia-inducible factor- $1 \alpha$ pathways $[\mathrm{J}]$. Hepatology, 2011, 53(1): 181-192.

[41] LUO T, FU J, XU A, et al. PSMD10/gankyrin induces autophagy to promote tumor progression through cytoplasmic interaction with ATG7 and nuclear transactivation of ATG7 expression $[\mathrm{J}]$. Autophagy, 2016, 12(8): 1355-1371.

[42] YANG C, TAN Y X, YANG G Z, et al. Gankyrin has an antioxidative role through the feedback regulation of Nrf2 in hepatocellular carcinoma $[J]$. J Exp Med, 2016, 213(5): 859-875.

[43 ] LIU R, LI Y, TIAN L, et al. Gankyrin drives metabolic reprogramming to promote tumorigenesis, metastasis and drug resistance through activating $\beta$-catenin/cMyc signaling in human hepatocellular carcinoma[J]. Cancer Lett, 2019, 443: 34-46.

[44] VANDER HEIDEN M G, DEBERARDINIS R J. Understanding the intersections between metabolism and cancer biology $[\mathrm{J}]$. Cell, 2017, 168(4): 657-669.

[45] HOWELL L A, PETERSON A K, TOMKO JR. R J. Proteasome subunit $\alpha 1$ overexpression preferentially drives canonical proteasome biogenesis and enhances stress tolerance in yeast [J]. Sci Rep, 2019, 9(1): 12418.

[46] RUT W, DRAG M. Human 20S proteasome activity towards fluorogenic peptides of various chain lengths $[\mathrm{J}]$. Biol Chem, 2016, 397(9): 921-926.

[47] DIKIC I. Proteasomal and autophagic degradation systems[J]. Annu Rev Biochem, 2017, 86(1): 193-224.

[48] QIN J, LONG B, LUO L, et al. Identification of proteasome subunit alpha type-1 as a novel biomarker in $\mathrm{HBV}$-associated hepatocellular carcinoma tissue interstitial fluid by proteomic analysis $[\mathbf{J}]$. Int J Clin Exp Pathol, 2017, 10(7): 7812-7820.

[49] TAN Y, QIN S, HOU X, et al. Proteomic-based analysis for identification of proteins involved in 5fluorouracil resistance in hepatocellular carcinoma[J]. Curr Pharm Des, 2014, 20(1): 81-87.

[50] BAIZ D, POZZATO G, DAPAS B, et al. Bortezomib arrests the proliferation of hepatocellular carcinoma cells HepG2 and JHH6 by differentially affecting E2F1, p21 and p27 levels[J]. Biochimie, 2009, 91(3): 373-382.

[51] HUANG I T, DHUNGEL B, SHRESTHA R, et al. Spotlight on Bortezomib: potential in the treatment of hepatocellular carcinoma $[\mathrm{J}]$. Expert Opin Investig Drugs, 2019, 28(1): 7-18.

[52] AUGELLO G, MODICA M, AZZOLINA A, et al. Preclinical evaluation of antitumor activity of the proteasome inhibitor MLN2238 (ixazomib) in hepatocellular carcinoma cells $[\mathrm{J}]$. Cell Death Dis, 2018, 9(2): 28.

[53] VANDEWYNCKEL Y P, COUCKE C, LAUKENS D, et al. Next-generation proteasome inhibitor oprozomib synergizes with modulators of the unfolded protein response to suppress hepatocellular carcinoma $[\mathrm{J}]$. Oncotarget, 2016, 7(23): 34988-35000.

[本文编辑 余 方 刘丽娜] 